\title{
Design and realization of indoor VLC-Wi-Fi hybrid network
}

\author{
Wentao Zhang, Li Chen*, Xiaohui Chen, Zihao Yu, Zhiyuan Li, Weidong Wang \\ Optical-Wireless Communication Key Lab of Chinese Academy of Sciences, \\ University of Science and Technology of China, Hefei 230027, China \\ * Corresponding author, Email: chenli87@ustc.edu.cn
}

\begin{abstract}
Indoor wireless communication networking has received significant attention along with the growth of indoor data traffic. VLC (Visible Light Communication) as a novel wireless communication technology with the advantages of a high data rate, license-free spectrum and safety provides a practical solution for the indoor high-speed transmission of large data traffic. However, limited coverage is an inherent feature of VLC. In this paper, we propose a novel hybrid VLC-Wi-Fi system that integrates multiple links to achieve an indoor high-speed wide-coverage network combined with multiple access, a multi-path transmission control protocol, mobility management and cell handover. Furthermore, we develop a hybrid network experiment platform, the experimental results of which show that the hybrid VLC-Wi-Fi network outperforms both single VLC and Wi-Fi networks with better coverage and greater network capacity.
\end{abstract}

Keywords: hybrid network, VLC, Wi-Fi, user access, mobility management, handover mechanism, multipath transmission

\section{Introduction}

Currently, $70 \%$ of wireless communication data traffic takes place indoors, and in the future, indoor traffic may approach $90 \%^{[1]}$. However, the indoor coverage of a wireless communication network is an issue remaining to be determined in the field of wireless communication. Accordingly, the indoor coverage of wireless networks is the major development of future networks. Cellular systems as wide area networks provides service for indoor users; however, a serious loss of wireless signals through walls makes it impossible to realize a high wireless transmission data rate. Therefore, small-scale base stations deployed indoors have received considerable attention.
An indoor femtocell base station improves the service quality to users. However, wireless resource allocation and interference management bring about high complexity to the network design. The deployment of WLAN node as a widely applied indoor wireless communication method also faces the challenge of wall blockage. Furthermore, the co-channel propagation of Wi-Fi for all users causes network congestion when there are too many users accessing the network.

VLC is a potentially effective wireless communication technology for supplementing RF (Radio Frequency) communication with the advantages of an abundant free spectrum, no electromagnetic interference, potential of high data rate, low cost and safety,

Manuscript received May 29, 2017; accepted Jul. 16, 2017

This work is supported by National Program on Key Basic Research Project of China (No. 2013CB329205), National Natural Science Foundation of China (No. 61601432) and Fundamental Research Funds for the Central Universities. 
etc. ${ }^{[2]}$. Downlink communication with a high data rate in homes and offices is a typical application scenarios of $\mathrm{VLC}^{[3]}$. However, the LOS (Line of Sight) communication characteristic of VLC results in two limitations: the coverage radius of VLC hotspot is generally less than $1 \mathrm{~m}$ and an optical link is easily interrupted from occlusions. Thus, the combination of a VLC and other indoor wireless communication technologies is a potential solution for the coverage of indoor wireless networks. As the most common indoor wireless network coverage technology available, Wi-Fi generally has a much larger coverage than VLC. The combination of VLC and Wi-Fi may therefore solve the problem of limited VLC coverage.

In this study an indoor VLC-Wi-Fi hybrid network for indoor wireless communication was designed. A hybrid network with a simple structure is easy to deploy and consists of two types of APs (Access Point): Wi-Fi AP and VLC AP. The designed VLC AP integrates VLC for a downlink and IR (Infrared) communication for an uplink, whereas the Wi-Fi AP adopted is a commercial product. Both APs are connected to the Internet through an Ethernet port. Terminal users can obtain services from two types of networks either individually or simultaneously. Moreover, transmission links including a VLC downlink, an IR uplink, a Wi-Fi downlink and a Wi-Fi uplink can be flexibly paired to a fullduplex link. The designed hybrid network integrates the respective advantages of $\mathrm{RF}$ communication and VLC, where the Wi-Fi AP provides wide coverage, the VLC AP supplies deep coverage for local region filled with traffic and both APs are connected to the Internet through the same network interface. We developed an experiment platform based on the designed hybrid network and analyzed the transmission performance based on the results of several experiments, including a vertical handover, horizontal handover, and multi-path transmission.

The rest of this paper is organized as follows. Section 2 reviews the VLC networking technology and integration with heterogeneous networks, and then the motivations and challenges of realizing a VLCWi-Fi hybrid network are presented. Section 3 de- scribes the key parts of designed network including the architecture of the hybrid network, the design of the VLC cells, the protocol of the hybrid network, access initialization and user mobility management mechanisms. The implementation of an experiment platform of the hybrid network and the analysis of the transmission are provided in section 4. Finally, section 5 gives some concluding remarks regarding this research.

\section{Related works}

VLC networking technology and its integration with heterogeneous networks have achieved substantive developments in recent years, including the structural design of a VLC network, duplexing and multiplexing, a VLC cell design, interference management and network handover mechanisms. Ref. [4] proposes a coalition formation for interference management in VLC networks, where VLC APs are designed to selforganize into cooperation coalitions based on the orthogonal time or frequency domain.

\subsection{VLC networking}

Duplexing is still a challenge despite the many schemes that have been proposed. Establishing a bidirection VLC link is theoretically feasible ${ }^{[5,6]}$, but remains impractical owing to excessive energy consumption at the user terminal devices and discomfort to the users' eyes. Wi-Fi used as uplink is a potential scheme $e^{[7,8]}$, however, a co-channel transmission limits the throughput. Another feasible scheme for achieving an uplink without electromagnetic interference is IR communication. Currently, the transmission rate of Fast Infrared is up to several tens of Mbit/s, whereas the power of an IR transceiver is only several tens of $\mathrm{mW}$, and thus IR communication can satisfy the requirements of a high uplink rate and low consumption. Ref. [9] compares various uplink options to demonstrate their advantages and disadvantages, and the use of near UV (Ultraviolet) and near IR LEDs for implementing a redundant uplink channel is recommended. However, similar to 
VLC, IR communication has a strong directivity and an obvious transmission performance gap exists between the VLC and IR, necessitating a specific design.

Multiple access is one of the key technologies of VLC networking. Various multiple access technologies of VLC networking, e.g. SDMA (Space Division Multiple Access), CDMA (Code Division Multiple Access), TDMA (Time Division Multiple Access) and FDMA (Frequency Division Multiple Access), have been studied. Ref. [10] proposes a VLC system adopting SDMA which can simultaneously serve multiple users in different positions, as shown through simulations, and SDMA can achieve a capacity several times higher than that of TDMA; however, this brings about high cost and complexity owing to the use of a special angle diversity transmitter. An optical CDMA system is presented in Refs. [11,12], where orthogonal codes are assigned to users. In a CDMA system, data sequences of multiple users are not completely orthogonal owing to the impact of a time-delay and multi-path fading resulting in MAI (Multiple Access Interference). MAI severely limits the communication capacity and performance of CDMA systems and it is difficult to eliminate in a VLC system. Ref. [13] presents VLC position system based on block encoding TDMA. In Refs. [14,15] a VLC uplink based on IFDMA (Interleaved Frequency Division Multiple Access) with a lower peak-to-average power ratio is demonstrated, which brings about an improved BER performance compared with OFDMA (Orthogonal Frequency Division Multiplexing Access). Ref. [16] proposes an experimental demonstration of VLC based on IDMA (Interleave Division Multiple Access), which offers better bit error rate performance compared with OFDMA. In addition, a novel multiple access technology for VLC is proposed in Ref. [17], and consists of NOMA (Non-Orthogonal Multiple Access) scheme and OFDMA with the advantages of high throughput, flexible bandwidth allocation and higher system capacity for a larger number of users. However, IDMA and NOMA-OFDMA greatly increase the complexity and cost of the system. In addition, the communication range of VLC is short, the coverage of a single AP is small, and indoor users are in general few in number and concentrated in small areas. Therefore, TDMA, which is easy to implement, is the optimal solution for realizing a practical hybrid network at low cost.

\subsection{VLC hybrid networking}

VLC-Wi-Fi hybrid networking can accomplish two goals: improving network throughout and increasing the coverage. A hybrid VLC and RF network is proposed in Refs. [18,19], in which VLC is used to provide a high system capacity, and RF is used to provide a wide coverage, and a network protocol combined with access, horizontal and vertical handover mechanisms is then applied. Research on maximizing the capacity of a VLC-WLAN hybrid network is discussed in Ref. [20], where multi-user dynamic resource allocation strategy is adopted. Design of hybrid network topology is also one of the key issues. In Ref. [21], RF-VLC HetNets are discussed based on a full system perspective, and three kinds of potential logical topologies are described. A symmetric VLC connection routes all traffic through the VLC $\mathrm{AP}$, whereas an asymmetric VLC connection routes the downlink through the VLC AP and uplink traffic through the RF AP. Depending on whether the uplink interferes with the RF connection, the symmetric network topology can be further divided into two types. Finally, a theoretical performance analysis of hybrid FSO/RF links is described in Ref. [22], where a link quality scheduling strategy is proposed.

\subsection{Motivations and challenges}

Current researches into VLC network have been mainly focused on theoretical analyses, and there has been a lack of practical network deployment solutions. To propose a practical hybrid VLC-Wi-Fi networking solution, the first thing to consider is reducing the deployment cost and the second is fitting the network to the characteristics of indoor scenarios. In terms of network deployment, we generally extend broadband network services into room and office use 


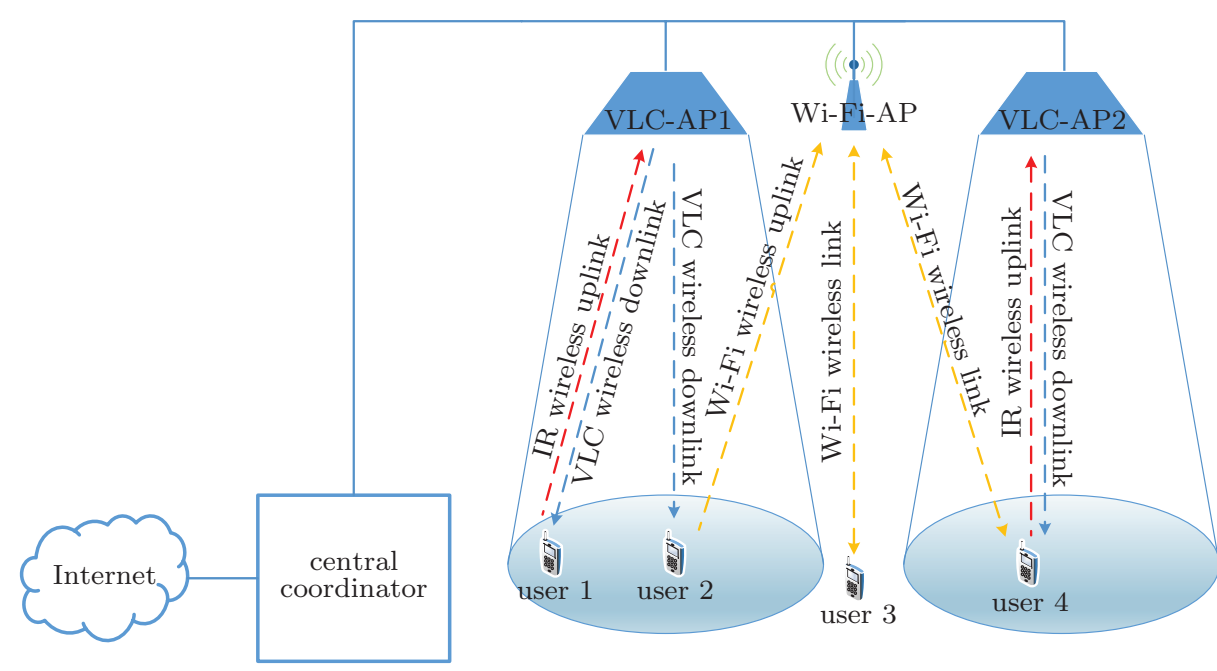

Figure 1 Hybrid VLC-Wi-Fi network model

by installing a Wi-Fi AP device or routing equipment. To meet the demands of easy integration with an indoor Wi-Fi network, convenient and flexible deployment, and expansion, the technical design of the VLC equipment can be based on Wi-Fi devices. Normally, indoor users with low mobility are irregularly distributed, and concentrated within the coverage of light. Indoor lighting will frequently be blocked by various obstacles, resulting in communication interruption; meanwhile, RF signals are significantly attenuated by the impact of such blocking, and therefore future indoor networks should mainly consider interruptions or degradations that occur from blocking. VLC is used to enhance the network service capabilities of partial areas where users are concentrated. In addition, users can gain diversity through the aggregation of VLC and RF links. A user terminal can access a network through multiple wireless APs simultaneously, and all types of links can be flexibly scheduled to provide a bi-directional transmission, and user data are aggregated at the network layers.

Herein, we focus on a practical and feasible indoor hybrid network deployment, although the details of link scheduling and handover are not discussed. The following aspects should be determined: the design of the VLC-Wi-Fi network architecture, the solution of duplex and multiple access, the design of a VLC multi-cell, multi-link end-to-end transmission, and an efficient mechanism for access control and mobility management.

\section{System model and design}

The VLC-Wi-Fi hybrid network model, which consists of a central coordinator, Wi-Fi AP, and VLC AP is shown in Fig. 1. Two types of APs are connected to the Internet through a central coordinator. As illustrated, user 1 accesses the VLC AP with a VLC downlink and IR uplink, user 2 accesses the VLC AP and Wi-Fi AP simultaneously with a VLC downlink and Wi-Fi uplink, user 3 accesses the WiFi AP with a Wi-Fi uplink and downlink, and user 4 accesses the VLC AP and Wi-Fi AP simultaneously with a VLC+Wi-Fi downlink and an IR+WiFi uplink. The fundamental functions of the central coordinator are shown in Fig. 2, including the information storage of the user access information and the link state information, user access management, slot allocation and link management. The link management module collects access information and link state information to schedule and control all transmission links. We define two work patterns: FULLLINKS and BACKUP. When a user terminal operates using a BACKUP pattern, the system allocates a proper link according to the user access and link 
state information, whereas the other available links remain as candidates. Once an active link breaks off because the lighting is blocked or when a user moves from one cell to another, the BACKUP pattern ensures a continuous transmission through an alternative link. When the user terminal operates using a FULL-LINKS pattern, the data are divided into two sub-flows transmitted through the VLC link and WiFi links, respectively, based on the MPTCP (MultiPath Transmission Control Protocol), which greatly increases the system bandwidth. When several links are stable, the total bandwidth reaches close to the sum of the bandwidths of each link. VLC multiple access mode uses time slot aloha and full network synchronization of the user terminals by receiving periodic broadcast beacon frames from the VLC AP. Multiple VLC cells reuse resources through time slot orthogonalization.

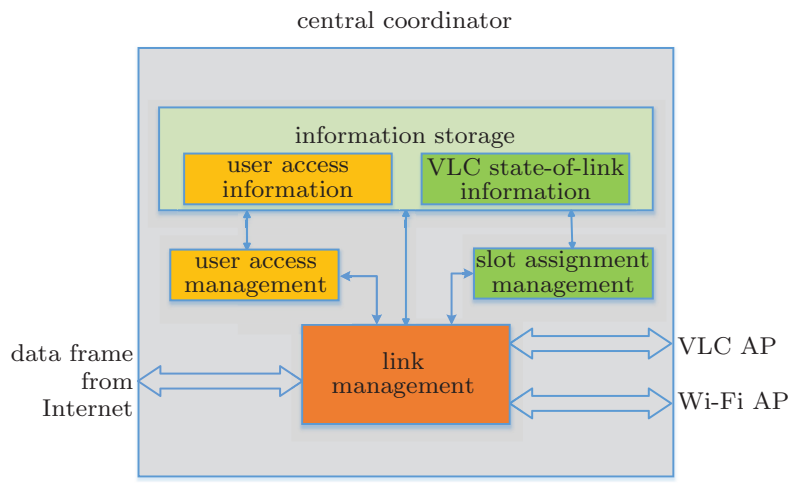

Figure 2 Fundamental function framework of central coordinator

\subsection{Structure of multiple cells}

The downlink of VLC AP adopts an OFDM-based visible light transmission, whereas the uplink adopts OFDM-based IR transmission. The diffusion angles of white-LED and IR-LED together determine the spatial service radius of the VLC AP. In contrast to traditional RF wireless communication, VLC has intense directivity which is beneficial in simplifying the VLC multi-cell structure. This paper describes the design of the time slot resource reuse structure of a multi-cell VLC, as shown in Fig. 3, where $S_{1}$ and $S_{2}$ are orthogonal time slot channels, $L_{1}$ is the distance between adjacent VLC APs and $L_{2}$ is the distance between diagonal VLC APs $\left(L_{2}=\sqrt{2} \cdot L_{1}\right)$. With such a structure, the interference between neighbor cells is eliminated by the time division, whereas the interference between diagonal cells is eliminated through spatial isolation. We assume that Wi-Fi has full coverage of the indoor space.

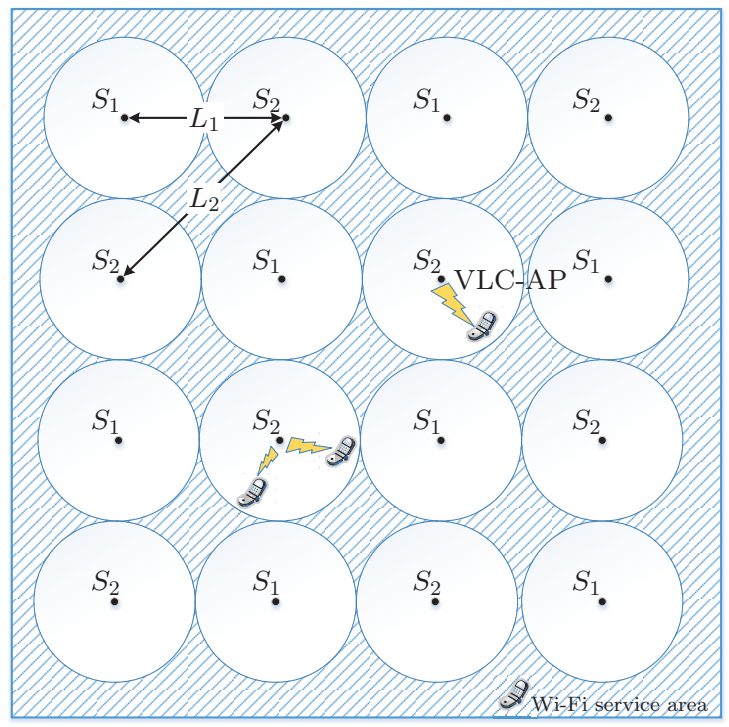

Figure 3 Structure diagram of hybrid network cells

\subsection{Protocol stack of hybrid network}

The protocol stack of a hybrid network is shown in Fig. 4. The downlink data frames separate at the network layer where different IP headers are added to the frames transmitted through different physical links. After being processed at the MAC and PHY layers, the frames transmitted over the Wi-Fi link are sent to users through the Wi-Fi AP, whereas the frames transmitted over the VLC link are first sent to Tx-PNC and then modulated to LED lamps. RxDEV receives and analyzes the physical frames from an optical link which are then combined into MAC frames. The MAC frames are transmitted to the user through an Ethernet port. The user receives the data frames over a Wi-Fi link through a Wi-Fi wireless card. The process at the user terminal (e.g., removing the IP header, analysing the port) for all the received data frames of the VLC and Wi-Fi is applied 


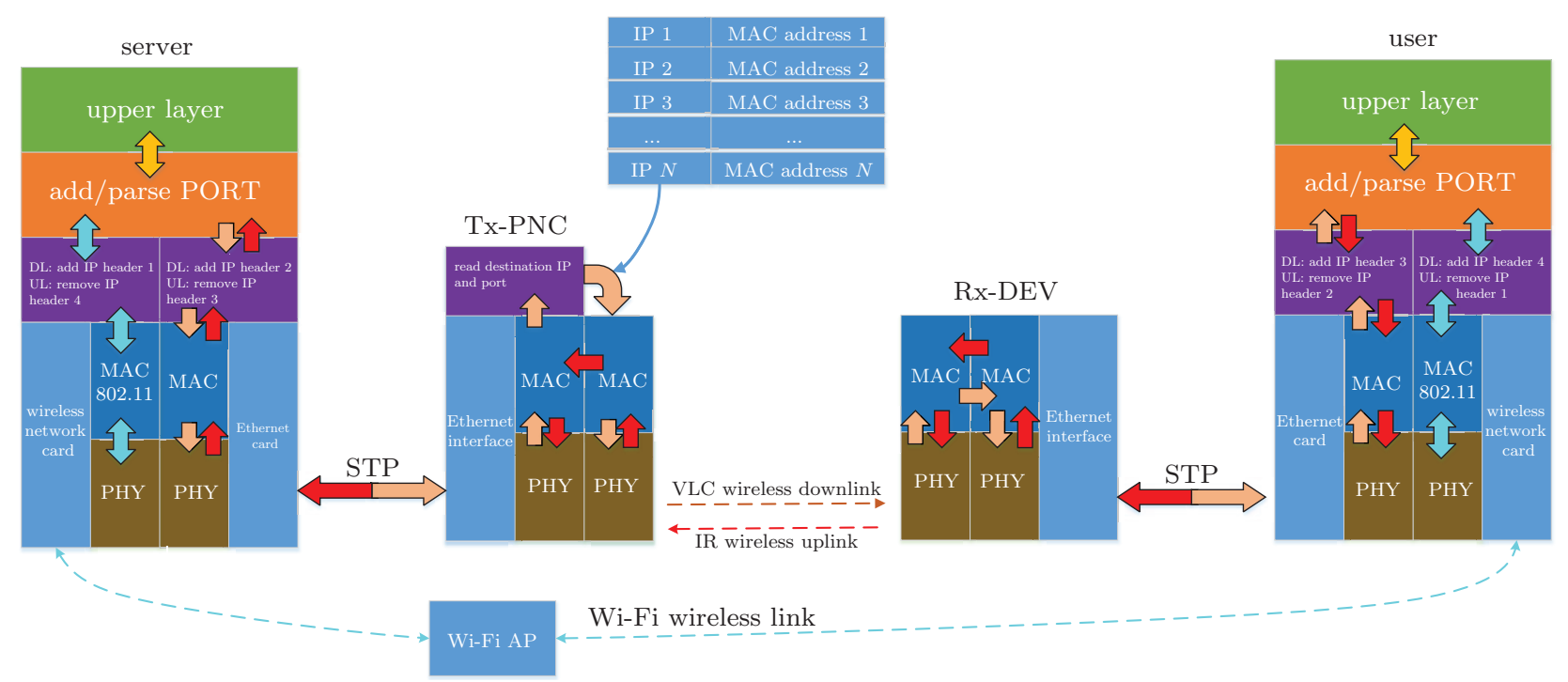

Figure 4 Hybrid network protocol stack

from the lower layer to the upper layer, contrary to the process applied at the server. The upstream data frames are also branched at the network layer, and the transmission of the upstream frames is similar to that of the downstream frames, however, the infrared upstream frames include a signaling frame CAP (Contention Access Period), which is generated under certain conditions (see Fig. 5) at the Rx-DEV. According to the CAP frame feedback of the user's slot and location information used to determine the data frame transmission time and channel is the key to realizing multi-user optical link access.

The TDMA-based optical link MAC layer protocol enables multiple terminals or nodes to simultaneously communicate through multiple access. In this paper, the design of a dedicated optical link MAC layer protocol with reference to 802.15 .3 is described. The MAC layer frameworks of Tx-PNC and Rx-DEV are shown in Fig. 5. The physical frames include the VLC downstream and IR upstream physical frames. A VLC downstream physical frame includes a beacon frame and a data frame. A beacon frame is the starting frame of the VLC downlink super-frame, whose main function is to characterize the starting position and the number of data frames of each user in the current super-frame. An IR uplink physical frame includes a CAP frame and data frame, where a CAP frame is a signaling frame used to feed the user's location information, adjust the type of business and size of the time slot. The user sends a CAP frame under the following three conditions: (1) when moving from one VLC AP coverage to another; (2) when changing the type of business; and (3) when remaining in the coverage of a VLC AP, and when the counter times out. The server of the system obtains the cell-id and user-id by analyzing the received CAP frame, and the user's location information is then known and recorded. In Tx-PNC, MAC frames received through an Ethernet port are analyzed and separated into shorter physical frames which will be sent to different buffers according to the mapping table; finally the data frames in the buffers are sent to the corresponding channel based on the user location information obtained from the CAP frame. In contrast, the uplink physical frames are first sent to different buffers based on the user-id, and then are combined into longer MAC frames, which will be transmitted to the Ethernet port. With Rx-DEV, we need to analyze the received physical frame to determine whether it is a beacon frame, if so, conduct a further analysis to obtain the user's time slot information and beacon-id. If the beacon-id changes, which indicates that the user's location has changed, an upstream CAP frame will be generated and sent immediately. The upstream data and CAP frames are sent through the same channel. 


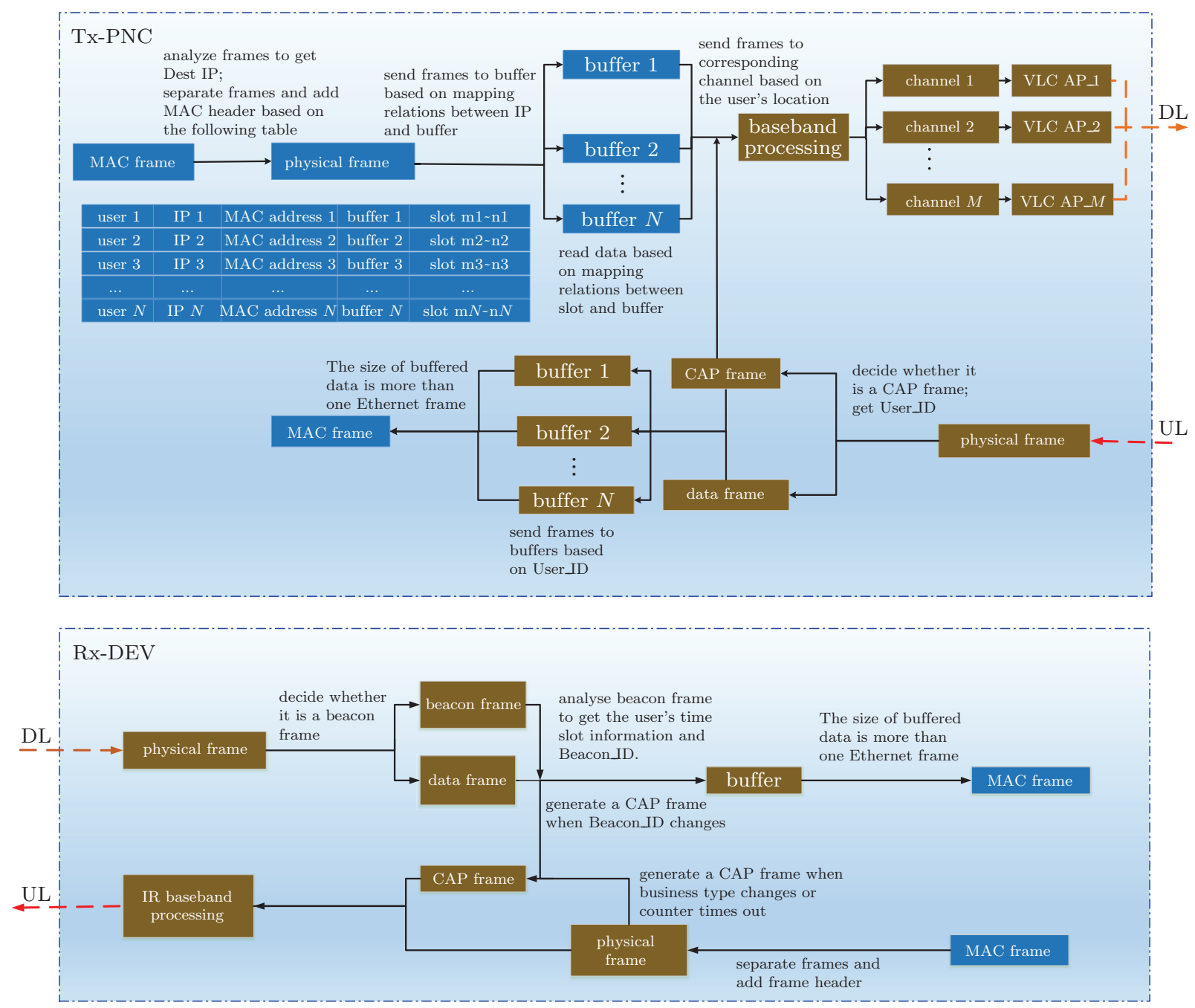

Figure 5 MAC layer framework of Tx-PNC and Rx-DEV

\subsection{User initialize access}

The user initialization access shown in Fig. 6 mainly accomplishes user registration and link resource allocation. There are two scenarios for a hybrid network to provide user service. In one case, the user is covered by a Wi-Fi AP, but can not be covered by a VLC AP. When the user can not receive a broadcast beacon frame from the VLC AP, the user terminal accesses the Wi-Fi AP and completes the sub-net registration; meanwhile the central coordinator records the user access information, and the hybrid network provides users with communication services through the Wi-Fi sub-net. In the other case, a user simultaneously covered by both a Wi-Fi AP and VLC AP needs to register on two sub-nets. The central coordinator handles the user requests based on the utilization state of the cell resources: i.e., either a WiFi sub-net or a VLC sub-net is selected to serve the user, or both sub-nets provide service together. As a special case, when an IR wireless uplink is interrupted, the user can finish the VLC sub-net registration through a Wi-Fi uplink.

\subsection{Mobility management and handover}

A cell or sub-net handover is caused as a result of user movement. Because Wi-Fi continuously covers the users, a handover mainly takes place between VLC cells. A handover can be divided into three typical cases: when the user moves from one VLC 


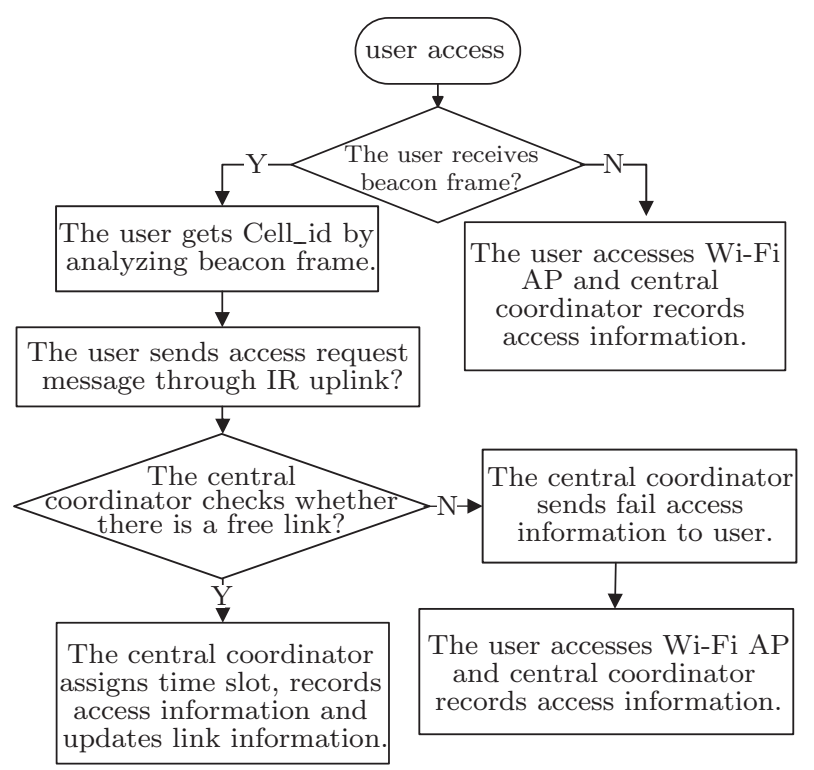

Figure 6 Fundamental function framework of central coordinator

cell to another; when the user moves from a VLC cell to outside the VLC cell; when a user moves from outside a VLC cell to inside the VLC cell. The first case is a horizontal handover between VLC cells, and the last two cases are a vertical handover between a VLC cell and a Wi-Fi cell. We designed a simple handover mechanism based on the user location for VLC cells with a horizontal handover in which the user terminal periodically transmits its access information through an uplink, and once the access information changes, the central coordinator immediately migrates the user data to a new cell. With a handover, the transmitter periodically transmits a beacon frame at the beginning of each super frame, and users receive and analyze this frame to obtain a cell-id. The user terminal then generates and transmits a CAP frame including the cell-id and user-id to the transmitter, and the central coordinator then records and updates the user's access information by analyzing the uplink CAP frame, and once the cellid changes, the transmitter immediately migrates the data to a reallocated VLC link. A vertical network handover occurs when the user's feedback of the access information is lost or reappears at the central coordinator, and in practical terms, vertical handover is based on the path management of the MPTCP.

\section{Experiment evaluation}

\subsection{Experiment platform}

To evaluate the function and performance of the designed VLC-Wi-Fi hybrid network, we set up an experiment system platform, which is shown in Fig. 7.

Fig. 8 shows the actual hybrid network where a proxy server and the central coordinator are placed on the same PC. The central coordinator consists of a PC and an FPGA board, i.e., a Tx-PNC, wherein the $\mathrm{PC}$ is used as the information storage module, which can obtain the user's access information by analyzing the infrared uplink CAP frame or the Wi-Fi uplink data frame. The FPGA board has two data channels designed to correspond to cells 1 and 2 respectively, where the slots of the two cells are orthogonal. The two cells periodically send a beacon frame. In addition, the MAC layer protocol of the optical link is implemented on the FPGA board which is connected with the server through the network interface, and transmits the data frame to the corresponding cell according to the user's access information. The data that have been processed through the baseband, DAC and driver circuit will be transmitted through the LED lamp. At the user terminal, a photoelectric conversion and amplification processing are first conducted on the modulated optical signals received by the APD (Avalanche Photo Diode). The signals are then processed and combined into MAC frames on the FPGA board, and finally the MAC frames are sent to the user's PC through an Ethernet interface. The FPGA board used is a Xilinx Virtex7 series VC707 development board. OFDM is used as the modulation method of the baseband. The VLC AP consists of four LEDs and an infrared transceiver. The bandwidth of the LED is $6 \mathrm{MHz}$, and the driving voltage and current of the LED are $30 \mathrm{~V}$ and 300 $\mathrm{mA}$, respectively. The receiving terminal consists of an APD (C12702-11), an IR transceiver, an FPGA board and a Wi-Fi wireless card. The primary experiment parameters are shown in Tab. 1.

In a conventional cellular network, a problem occurs in the signal strength attenuation at the cell 


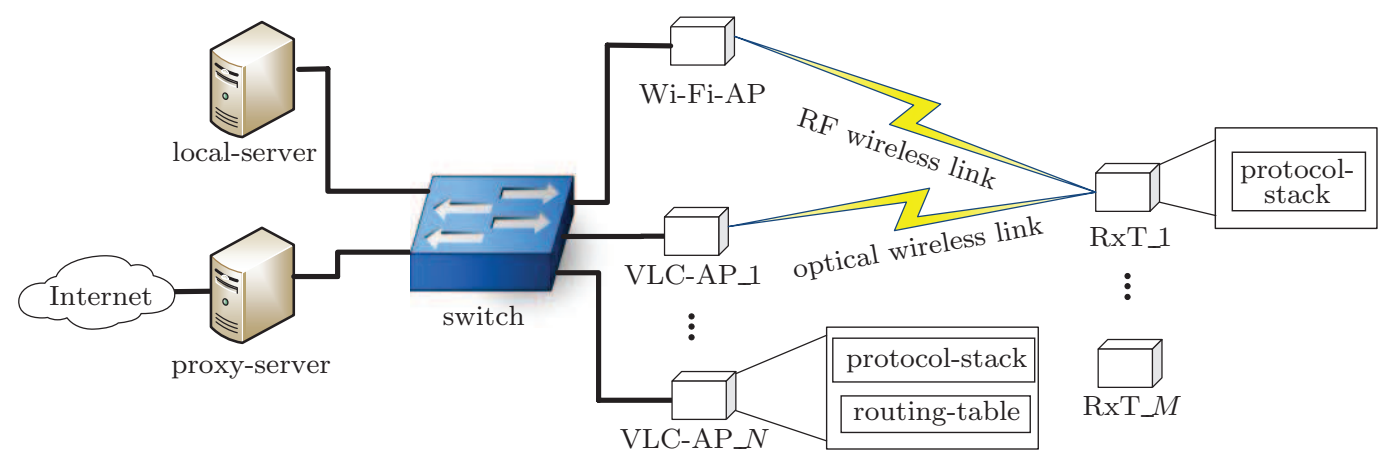

Figure 7 Structure of hybrid VLC-Wi-Fi experiment platform

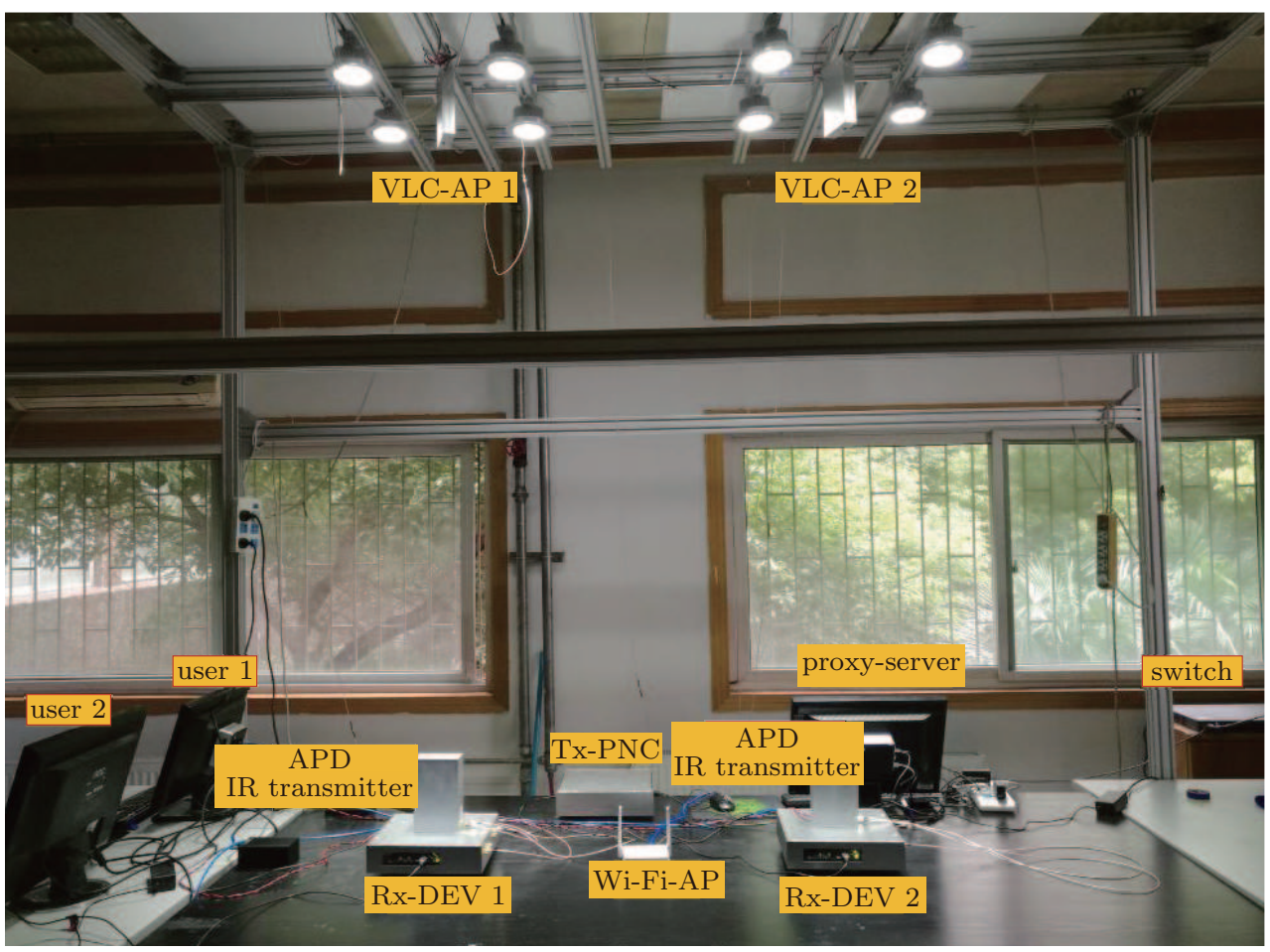

Figure 8 Hybrid VLC-Wi-Fi experiment platform

Table 1 Experiment parameters

\begin{tabular}{cc|cc}
\hline parameter & value & parameter & value \\
\hline vertical height & $2 \mathrm{~m}$ & bias voltage of IR-Rx & $20 \mathrm{~V}$ \\
cells spacing & $1.2 \mathrm{~m}$ & bias voltage of LED & $30 \mathrm{~V}$ \\
LED $-10 \mathrm{~dB}$ bandwidth & $<3 \mathrm{MHz}$ & LED transmit power & $0.9 \mathrm{~W}$ \\
LED modulation depth & $16 \%$ & LED FOV & $38^{\circ}$ \\
APD active area & $0.78 \mathrm{~mm}^{2}$ & IR-Rx FOV & $40^{\circ}$ \\
APD responsivity & $-2.5 \times 10^{4} \mathrm{~V} / \mathrm{W}$ & APD bandwidth & $100 \mathrm{MHz}$ \\
\hline
\end{tabular}

edge is quite server. To avoid the same problem in the VLC cells, we designed the VLC AP specifically, the details of which are shown in Fig. 9. When the vertical distance from the LED lamp to the floor is $1.8 \mathrm{~m}$, the coverage of the LED lighting is circular with a radius of $0.6 \mathrm{~m}$. We utilize four LEDs 


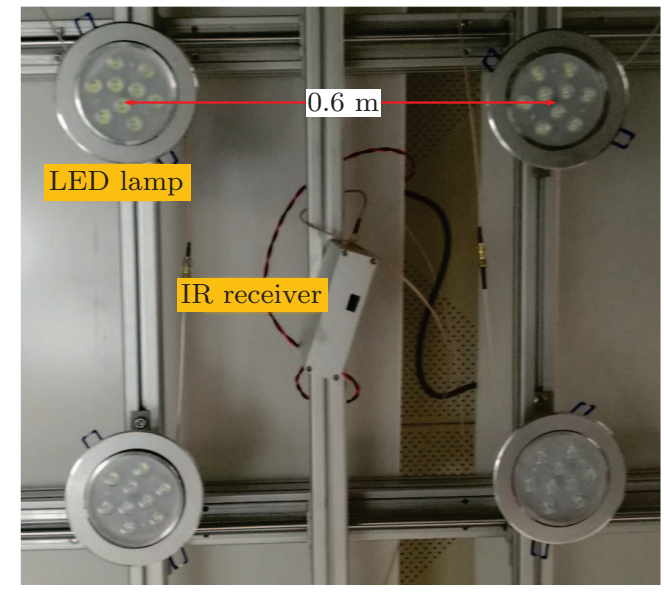

Figure 9 Structure of VLC AP with four LED lamps and one IR receiver

to form a square with a side length of $0.6 \mathrm{~m}$, allowing a radius of coverage of about $2 \mathrm{~m}$. An IR transceiver with similar coverage as a single LED is placed at the center of the VLC AP. The IR LED has a strong directivity in that the energy is concentrated within the diffusion angle, and thus the signal will not be significantly attenuated within the range of the IR transceiver coverage; however, the signal strength degrades sharply outside the coverage of the IR LED. At the intersection of the IR and LED coverage, the downlink optical signal strength will not be significantly attenuated, and thus the coverage of the designed has an overlapping area where the SNR of both the uplink and downlink reception can remain high, thereby guaranteeing quality communication when the user moves within the VLC cell. Fig. 10 shows the performance of the VLC cell

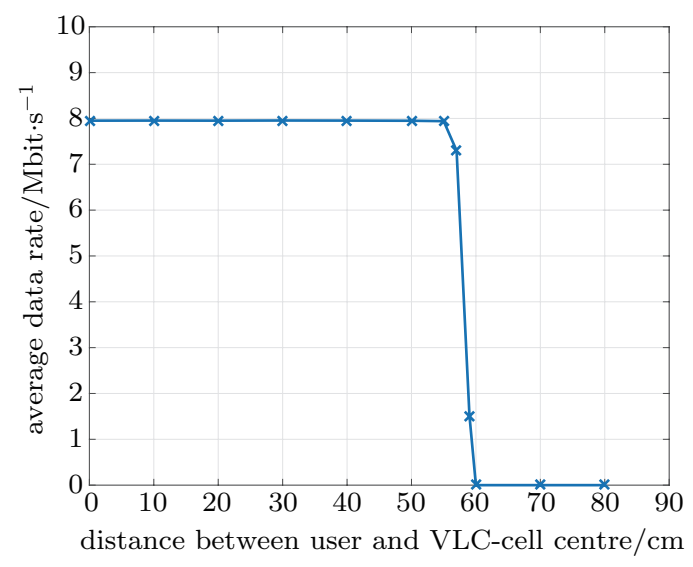

Figure 10 Coverage performance of single VLC AP coverage: when the user remains in a VLC cell, the average data rate is almost constant; however, once the user moves out of the VLC cell, the average rate drops to zero.

\subsection{Experimental results and analysis}

\subsubsection{Multi-link parallel transmission}

Utilization of the MPTCP can not only achieve link scheduling, it also greatly enhances the network capacity. We set the user terminal at the center of the VLC cell, and then carried out a series of experiments using a single VLC link, a single Wi-Fi link and VLC and Wi-Fi links combined, respectively. In detail, we transmitted three files with different types and sizes from the server to the user. The experiment results shown in Fig. 11 indicate that a multi-link parallel transmission provides a significant capacity gain, and the average data rate is close to the sum of the two links.

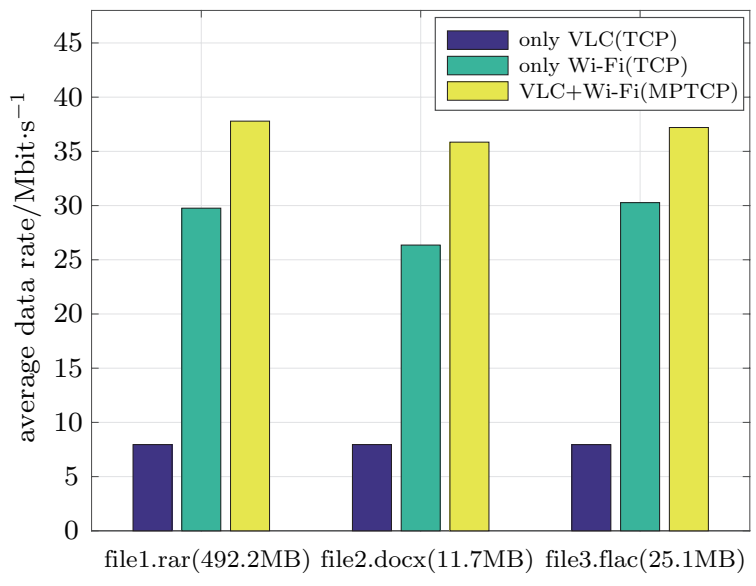

Figure 11 Performance of multi-link parallel transmission

\subsubsection{Mobility management and handover}

We designed the following experiment scheme, which is also shown in Fig. 12(a). The initial positions of both users 1 and 2 is source A, and the users then move across VLC cell 1, finally arriving at position $\mathrm{B}$ along the blue path. The initial positions of users 3 and 4 is source C, and the users then move across VLC cell 1 and VLC cell 2 sequentially, arriving at destination $\mathrm{D}$ along the green path. Users 1 and 3 use a BACKUP pattern in which the VLC is used as 
the main link and Wi-Fi is used as the backup link, whereas users 2 and 4 use a FULL-LINKS pattern. The speed of the users is set at around $0.1 \mathrm{~m} / \mathrm{s}$. The experiment results are shown in Fig. 12(b). At the source, all users access the network through a Wi-Fi link, and therefore data rate of each user is around $28 \mathrm{Mb} / \mathrm{s}$. When users 1 and 2 leave $\mathrm{A}$, a vertical handover from Wi-Fi to VLC occurs at $40 \mathrm{~cm}$. Because different patterns are used, there is an obvious data rate gap between user 1 and user 2. A vertical handover from VLC to Wi-Fi occurs when users 1 and 2 leave the VLC AP1 coverage. A horizontal handover between VLC cells occurs when users 3 and 4 move from VLC cell 1 to VLC cell 2, that is, a distance of

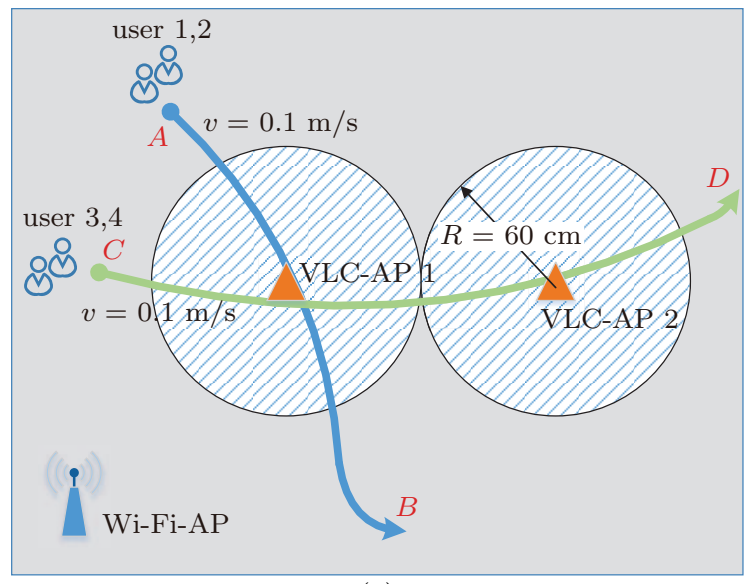

(a)

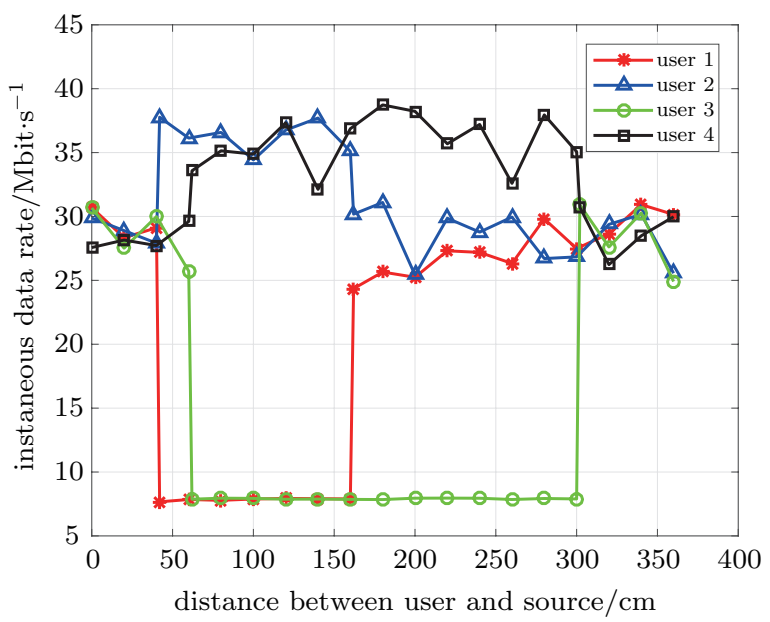

(b)

Figure 12 Mobility management and handover: (a) experiment scheme; (b) experiment results
$180 \mathrm{~cm}$, as shown in the figure, although the access information of users 3 and 4 changes from VLC cell 1 to VLC cell 2, the data rate is not decreased. Although the instantaneous rate of Wi-Fi changes clearly and frequently, the curves in the figure statistically indicate that the hybrid network can provide quality service for indoor users.

\section{Conclusion}

For this study, we designed and realized a hybrid VLC-Wi-Fi network, consisting of several VLC APs and one Wi-Fi AP. Both VLC and Wi-Fi APs are linked to the Internet through a central coordinator, whose fundamental functions are information storage, link management, user access, and slot assignment. In this hybrid network, there are multiple links available for scheduling. When a user is within VLC coverage, for a reliable high-speed transmission, the user can access the network through either an optical or RF channel, or through both optical and RF channels simultaneously. When a user is outside of VLC coverage, the user accesses the network through Wi-Fi. We also presented an extensible deployment scheme with a rectangular area by referring to a cellular network. TDM was adopted to eliminate inter-cell interference of the VLC, and an advanced resource allocation strategy needs to be further explored to improve the resource utilization. A hybrid network protocol combined with the transmission protocol of MAC and PHY layers, user access, mobility management, and a handover mechanism was proposed. In addition, we constructed an experiment platform based on the design of the hybrid network, and the experiment results show that the hybrid VLC-Wi-Fi network outperforms both single VLC and Wi-Fi networks, with better coverage and greater network capacity.

\section{References}

[1] S. Z. Chen, J. Zhao. The requirements, challenges, and technologies for $5 \mathrm{G}$ of terrestrial mobile telecommunication [J]. IEEE communications magazine, 2014, 52(5): $36-43$. 
[2] D. Karunatilaka, F. Zafar, V. Kalavally, et al. LED based indoor visible light communications: state of the art [J]. IEEE communications surveys \& tutorials, 2015, 17(3): 1649-1678.

[3] A. Jovicic, J. Li, T. Richardson. Visible light communication: opportunities, challenges and the path to market [J]. IEEE communications magazine, 2013, 51(12): 2632.

[4] L. Chen, W. D. Wang, C. Zhang. Coalition formation for interference management in visible light communication networks $[\mathrm{J}]$. IEEE transactions on vehicular technology, 2017, 66(8): 7278-7285

[5] Y. Q. Wang, Y. G. Wang, N. Chi, et al. Demonstration of $575-\mathrm{Mb} / \mathrm{s}$ downlink and $225-\mathrm{Mb} / \mathrm{s}$ uplink bidirectional SCM-WDM visible light communication using RGB LED and phosphor-based LED [J]. Optics express, 2013, 21(1): 1203-1208.

[6] Y. F. Liu, C. H. Yeh, C. W. Chow, et al. Demonstration of bi-directional LED visible light communication using TDD traffic with mitigation of reflection interference $[\mathrm{J}]$. Optics express, 2012, 20(21): 23019-23024.

[7] S. H. Shao, A. Khreishah, M. B. Rahaim, et al. An indoor hybrid Wi-Fi-VLC internet access system [C]//IEEE 11th International Conference on Mobile Ad Hoc and Sensor Systems (MASS), 2014: 569-574.

[8] Z. T. Huang, Y. F. Ji. Design and demonstration of room division multiplexing-based hybrid VLC network [J]. Chinese optics letters, 2013, 11(6): 060603.

[9] M. Vladescu, D. T. Vuza. Redundant uplink optical channel for visible light communication systems [C]//Advanced Topics in Optoelectronics, Microelectronics, and Nanotechnologies VII, 2015: 92581J.

[10] Z. Chen, H. Haas. Space division multiple access in visible light communications [C]//2015 IEEE International Conference on Communications (ICC), 2015: 5115 C5119.

[11] M. F. Guerra-Medina, B. Rojas-Guillama, O. González, et al. Experimental optical code-division multiple access system for visible light communications [C]//IEEE 2011 Wireless Telecommunications Symposium (WTS), 2011: 1-6.

[12] M. Noshad, M. Brandt-Pearce. High-speed visible light indoor networks based on optical orthogonal codes and combinatorial designs [C]//2013 IEEE Global Communications Conference (GLOBECOM), 2013: 2436-2441.

[13] Y. N. Hou, S. L. Xiao, H. F. Zheng, et al. Multiple access scheme based on block encoding time division multiplexing in an indoor positioning system using visible light $[J]$. Journal of optical communications and networking, 2015, 7(5): 489-495.

[14] B. J. Lin, H. Yang, W. P. Ye, et al. Experimental demonstration of block interleaved frequency division multiple access for bidirectional visible light communications [J]. Optical engineering, 2017, 56(1): 016112016112 .
[15] B. J. Lin, X. Tang, H. Yang, et al. Experimental demonstration of IFDMA for uplink visible light communication [J]. IEEE photonics technology letters, 2016, 28(20): 2218-2220.

[16] B. J. Lin, W. P. Ye, X. Tang, et al. Experimental demonstration of interleave division multiple access visible light communication [J]. Optical engineering, 2017, 56(5): 056101.

[17] B. J. Lin, W. P. Ye, X. Tang, et al. Experimental demonstration of bidirectional NOMA-OFDMA visible light communications [J]. Optics express, 2017, 25(4): 4348-4355.

[18] D. A. Basnayaka, H. Haas. Hybrid RF and VLC systems: improving user data rate performance of VLC systems [C]//2015 IEEE 81st Vehicular Technology Conference (VTC Spring), 2015: 1-5.

[19] X. Bao, X. R. Zhu, T. C. Song, et al. Protocol design and capacity analysis in hybrid network of visible light communication and OFDMA systems [J]. IEEE transactions on vehicular technology, 2014, 63(4): 1770-1778.

[20] M. B. Rahaim, A. M. Vegni, T. D. Little. A hybrid radio frequency and broadcast visible light communication system [C]//2011 IEEE GLOBECOM Workshops (GC Wkshps), 2011: 792-796.

[21] T. D. Little, M. Rahaim. Network topologies for mixed RF-VLC HetNets [C]//IEEE 2015 Summer Topicals Meeting Series (SUM), 2015: 163-164.

[22] L. Chen, W. D. Wang, C. Zhang. Multiuser diversity over parallel and hybrid FSO/RF links and its performance analysis $[\mathrm{J}]$. IEEE photonics journal, 2016, 8(3): $1-9$.

\section{About the authors}

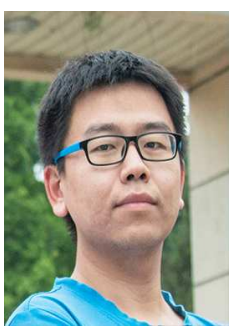

Wentao Zhang was born in Datong City, Shanxi Province. He received his B.E. degree in electronic engineering from South China University of Technology in 2015. He is now a Ph.D. candidate at the University of Science and Technology of China. His research interests include VLC, MIMO communication, and mmWave communication. (Email: zwentao@mail.ustc.edu.cn)

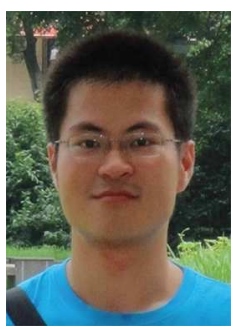

Li Chen [corresponding author] attended Harbin Institute of Technology (HIT) and obtained his B.S. degree in electrical and information engineering in 2009. He obtained his Ph.D. degree in electrical engineering from University of Science and Technology of China(USTC), Hefei, in 2014. In the same year, he won the Chinese Academy of Sciences President Award. He joined 
the USTC as a postdoctor from 2014 to 2016 . He is currently a faculty member of the USTC. (Email: chenli87@ustc.edu.cn)

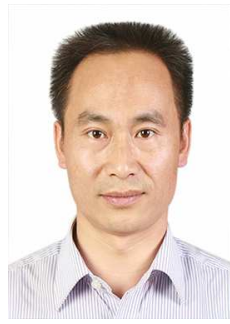

Xiaohui Chen was born in 1976. He received his B.S. and M.S. degrees in communication and information engineering from the University of Science and Technology of China(USTC), Hefei, in 1998 and 2004, respectively. He is currently an associate professor at the Department of Electronic Engineering and Information System, USTC. His current research interests include wireless network QoS, mobile computing, MAC protocol and VLC hybrid networking. (Email: cxh@ustc.edu.cn)

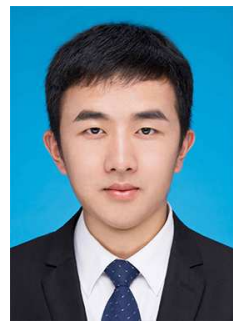

Zihao Yu received his B.S. degree from the University of Science and Technology of China(USTC), Hefei, in 2014 and his M.S. degree from the same university in 2017. He is currently working at Huawei Technologies Co., Ltd. His major research interests include multiple access and multi-cell handover in wireless communication networks. (Email: Ustcer00@mail.ustc.edu.cn)

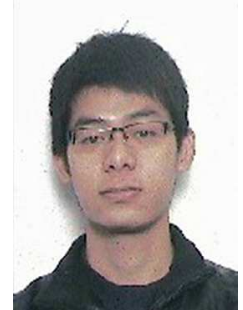

Zhiyuan Li attended the University of Electronical Science and Technology of China (UESTC), Chengdu, and obtained his B.S. degree in the School of Electronic Engineering in 2014. He is currently pursuing his Ph.D. degree in Department of Electronic Engineering and Information Science (EEIS), at USTC. His research interests include $5 \mathrm{G}$ millimeter wave mobile networks, stochastic geometry, and visible light communications. (Email: lzy886@mail.ustc.edu.cn)

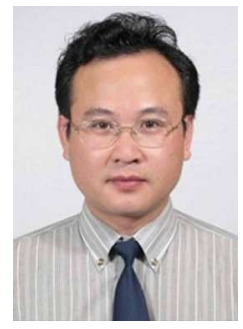

Weidong Wang received his B.S. degree from Beijing University of Aeronautics and Astronautics, China in 1989, and his M.S. degree from the University of Science and Technology of China(USTC), Hefei, in 1993. He is currently a full professor in the Department of Electronic Engineering and Information Systems, at USTC. His research interests include wireless communication, microwave, mmWave, and radar technologies. (Email: wdwang@ustc.edu.cn) 